\title{
Choking conditions for nonuniform viscous flows
}

\author{
F. J. Higuera and A. Liñán
}

\begin{abstract}
The laminar viscous compressible flow of a gas in a pipe of constant cross section joining two reservoirs is considered. As it is well known, the pipe flow becomes independent of the discharge pressure if this is lower than a critical value. Then it is found that the parabolic equations describing the high Reynolds number flow in the interior of the pipe tend to develop a singularity at the outlet, where the limiting temperature and velocity profiles satisfy a criticality condition. The structure of the nonuniform flow near the outlet consists of an effectively inviscid vortical bulk region and a viscous boundary layer of low velocity, whose thickness tends to zero at the outlet. Matching between these two regions determines the strength of the singularity. This two-layered structure is thought to be generic for nonuniform viscous flows having to satisfy a no-slip'boundary condition; in particular, the thin viscous layer is universal.
\end{abstract}

Consider the discharge of a gas from a large reservoir at pressure $P_{0}$ to a second expanse of gas at pressure $P_{e}<P_{0}$ through a pipe of length $L$ and radius $a$. Several regions can be distinguished in this flow for high values of the Reynolds number. There is first the region around the pipe mouthpiece, of characteristic length $a$, where the flow speeds up in an effectively inviscid manner (except in the thin boundary layer that begins growing on the pipe wall). Then the flow becomes quasi-one-directional as it progresses down the pipe. Transport effects will influence appreciably this flow, which we suppose to be laminar, when $a / L=O(\lambda / a)<1, \lambda$ being the mean-free path of the gas in the reservoir. The effect of the viscosity changes the shape of the velocity profile and leads to a decrease of the pressure. In the absence of heat losses through the walls, the mean density and temperature of the gas across the section of the pipe also decrease, whereas the mean velocity and Mach number increase. These changes are more pronounced as the pressure ratio $P_{e} / P_{0}$ decreases, until, for a certain $P_{e} / P_{0}=p^{*}$ dependent on $\lambda L / a^{2}$, the flow in the pipe attains a limiting state (choked flow) that is not modified by further decreasing $P_{e} / P_{0}$. As for the inviscid counterpart, critical conditions, meaning that the flow becomes "effectively sonic" and upstream wave propagation -ceases to be possible, must exist at the outlet of the pipe when the flow becomes independent of the outer pressure (for a further decrease of $P_{e}$ would otherwise modify the flow, if upstream propagation were still possible). Finally, a third region of characteristic length $a$ exists around the pipe outlet when $P_{e} / P_{0} \leqslant p^{*}$. This is a region of effectively inviscid fully two-dimensional vortical flow, with coexisting subsonic and supersonic zones, which matches upstream to the incoming pipe flow and leaves a pressure equal to $P_{e}$ downstream. The flow in this region is very complex and dependent on the discharge pressure, but fortunately it needs not be analyzed in any detail to characterize the choking because the choked flow is independent of the downstream conditions (at least on the scale of the pipe length).

In the remainder of this Brief Communication, we de- scribe the choked flow in the bulk of the pipe, corresponding to the second of the aforesaid regions, and present an asymptotic analysis of the solution near the critical section.

The quasi-one-directional flow in the pipe obeys the mass, momentum, and energy conservation equations

$$
\begin{aligned}
\frac{\partial(\rho u)}{\partial x}+\frac{1}{r} \frac{\partial}{\partial r}(r \rho v) & =0, \\
\rho u \frac{\partial u}{\partial x}+\rho v \frac{\partial u}{\partial r}= & -\frac{1}{\gamma} \frac{d p}{d x}+\frac{1}{r} \frac{\partial}{\partial r}\left(r \frac{\partial u}{\partial r}\right), \\
\rho u \frac{\partial H}{\partial x}+\rho v \frac{\partial H}{\partial r}= & \frac{1}{\operatorname{Pr}} \frac{1}{r} \frac{\partial}{\partial r}\left(r \frac{\partial H}{\partial r}\right) \\
& +\frac{\operatorname{Pr}-1}{\operatorname{Pr}} \frac{\gamma-1}{\gamma} \frac{1}{r} \frac{\partial}{\partial r}\left(r \frac{\partial u^{2}}{\partial r}\right),
\end{aligned}
$$

with the boundary conditions

$$
\begin{aligned}
& p^{(\gamma-1) / \gamma}=T=1-\frac{\gamma-1}{2} u^{2} \\
&=\frac{1}{1+(\gamma-1) M^{2} / 2} \quad \text { at } x=0, \\
& \frac{\partial u}{\partial r}=v=\frac{\partial T}{\partial r}=0 \quad \text { at } r=0, \\
& u=v=\frac{\partial T}{\partial r}=0 \quad \text { at } r=1 .
\end{aligned}
$$

Here, $H=T+(\gamma-1) u^{2} / 2$ is the stagnation enthalpy and $p=\rho T$. The variables $(x, r, u, v, p, \rho, T)$ have been nondimensionalized with the scales $\left(a \operatorname{Re}, a, c_{0}, c_{0} / \operatorname{Re}, P_{0}, \rho_{0}, T_{0}\right)$, respectively, where the subindex 0 means conditions at the reservoir, $c_{0}=\sqrt{\gamma P_{0} / \rho_{0}}, \operatorname{Re}=\rho_{0} c_{0} a / \mu>1$ is the Reynolds number, $\operatorname{Pr}$ and $\gamma$ are the Prandtl number and ratio of specific heats of the gas, and the transport coefficients have been assumed to be constant. Pressure variations across the pipe are neglected. Condition (4) at the entrance of the pipe states the conservation of entropy and stagnation en- 


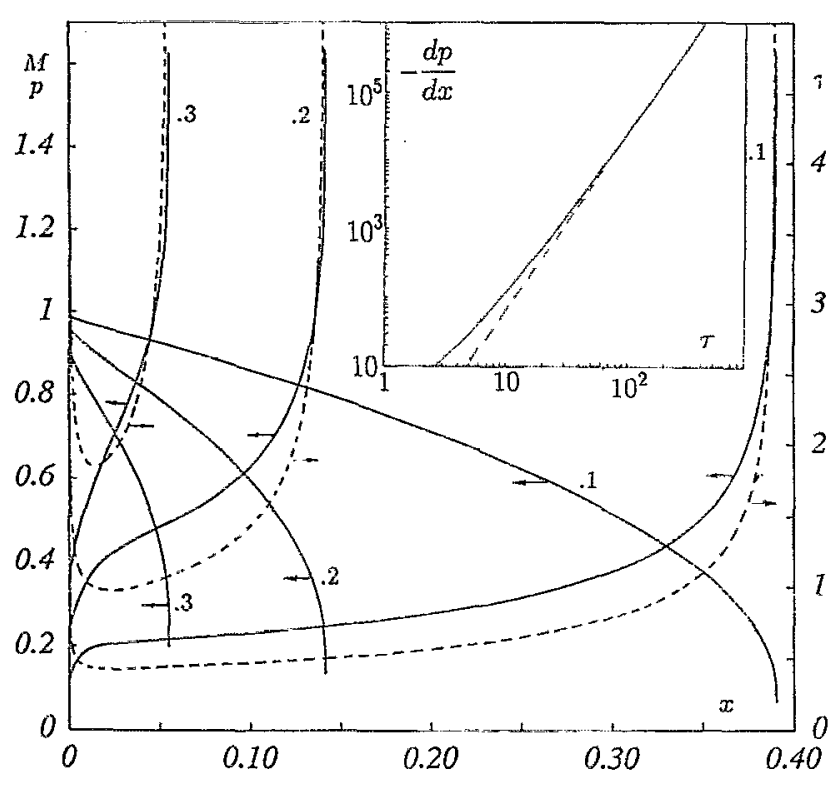

FIG. 1. Pressure (decreasing) and Mach along the axis (increasing) as functions of $x$ for $\operatorname{Pr}=1, \gamma=1.4$, and $M(0)=0.1,0.2$, and 0.3 . The dashed lines represent shear on the wall $\left(\tau=-\partial u /\left.\partial r\right|_{r=1}\right)$. Excerpt: logarithmic representation of pressure gradient versus shear near the critical section. The dashed line is the slope given by asymptotic theory.

thalpy in the mouthpiece, where transport effects are negligible. The two boundary conditions for the radial velocity $v$ determine the pressure.

Results of the numerical integration of (1)-(6), obtained by $\operatorname{Perez} z^{1}$ for $\operatorname{Pr}=1, \gamma=1.4$, and three different values of $M(0)$ are plotted in Fig. 1. As can be seen in this figure, the pressure decreases and the Mach number on the axis increases monotonically along the pipe down to a certain point $x=l[M(0)]$, where the curves develop infinite slopes. No solution of the problem exists beyond this point, so that $p(l)$ represents the minimum pressure attainable for the given $M(0)$, and the flow in the pipe becomes independent of the discharge pressure when $P_{e} / P_{0}$ decreases below $p(l)$. Hence $p(l)=p^{*}[M(0)]$ and the flow at $x=l$ is critical, in the aforesaid sense. The scaled shear stress $\left(\tau=-\partial u /\left.\partial r\right|_{r=1}\right)$ does also tend to infinity when $x>l$, and for $x \searrow 0$; this latter divergence being due to the initial development of the boundary layer, which starts growing at the entrance of the pipe and fills a sizable amount of the cross section for $x=O(1)$.

We now proceed to the asymptotic analysis of the solution of $(1)-(6)$ for $(l-x)<1$. Let $u_{l}(r)$ and $T_{l}(r)$ be the velocity and temperature distributions of the limiting flow at $x=l$ (i.e., immediately upstream of the region of twodimensional motion). These distributions depend to a large extent on the evolution of the flow all along the pipe [on $M(0)$ for the problem at hand], but must satisfy certain conditions discussed below. For flows with finite shear, a linear analysis of the evolution of small perturbations of wavelength short compared to the length of the pipe but large compared to its radius yields the necessary condition

$$
\lim _{r \rightarrow 1} u_{l}(r) \int_{0}^{r}\left(\frac{1}{M_{l}^{2}}-1\right) r d r=0
$$

for the flow $\left[u_{l}(r), T_{l}(r)\right]$ to be critical (see Refs. $2-4$ or the discussion below). Clearly, (7) cannot be fulfilled by a flow satisfying the no-slip condition $u_{l}(1)=0$. Hence attention must be turned to limiting flows with infinite shear: $u_{l} \simeq \alpha(1-r)^{\sigma}$ for $(1-r)<1$, for some $0<\sigma<1$. But, since a large pressure gradient is required to generate a large shear stress, and this pressure gradient affects to the whole section of the pipe, the flow near the outlet is subject to very strong accelerations, and thus transport effects are confined to a thin boundary layer near the walls.

We begin by considering the flow outside of this boundary layer. Here, the solution of (1)-(6) for ( $l$ $-x)<1$ is of the form

$$
\begin{aligned}
& p-p^{*}=(l-x)^{\delta} \bar{\pi}, \quad u-u_{l}=(l-x)^{\delta} U(r), \\
& v=(l-x)^{\delta-1} V(r), \quad T-T_{l}=(1-x)^{\delta} \Theta(r), \\
& \rho-\rho_{l}=(l-x)^{\delta} R(r),
\end{aligned}
$$

for some $0<\delta<1$ to be determined. The variables $(\bar{\pi}, U, V, \Theta, R)$ are the leading order of an asymptotic expansion for $(l-x)$ small, and $\rho_{l}=p^{*} / T_{l}$. Carrying these asymptotic expressions into the conservation equations (1)-(3) and the equation of state, and combining the resulting equations to eliminate $U, \Theta$, and $R$, we obtain the single equation

$$
\frac{d}{d r}\left(r \frac{V}{u_{l}}\right)+\frac{\delta \bar{\pi}}{\gamma p^{*}} r\left(\frac{1}{M_{l}^{2}}-1\right)=0
$$

for the radial velocity. In addition to the regularity condition $V=0$ at $r=0$, the solution of (9) must also satisfy a matching condition with the boundary layer near the wall, which is analyzed below. At first sight, such a condition overdetermines the problem, for there is only a first-order derivative in (9) (and $\bar{\pi}$ is merely a scale factor). In fact, the second boundary condition imposes a restriction on the limiting flow itself. We note that (9) does also describe the normal modes in an analysis of the response of the base flow $\left[u_{l}(r), T_{l}(r)\right]$, considered as a parallel flow, to small perturbations of the form $\Delta u=U(r) \exp [i k(x-c t)]$, etc., with $c=0$. Then $\delta$ must be identified with $-i k$. This shows explicitly that, if a solution of (9) exists satisfying the aforementioned conditions, the flow $\left[u_{l}(r), T_{l}(r)\right]$ is able to hold standing waves, being therefore critical.

For a base flow with finite shear at the wall ( $u_{l}^{\prime}$ finite), the second condition would be $V=0$ at $r=1$, and (7) would be recovered by integrating (9) from $r=0$, to $r=1$. On the other hand, for a flow with $u_{l} \simeq \alpha(1-r)^{\sigma}$ near the wall, Eq. (9) gives

$$
V=\frac{\delta \bar{\pi}}{\gamma p^{*}}\left(\frac{T_{w} / \alpha}{1-2 \sigma}(1-r)^{1-\sigma}-\alpha C(1-r)^{\sigma}\right),
$$

for $(1-r)<1$, where $T_{w}=T_{l}(1)$ and $C$ is an integration constant, equal to $\int_{0}^{1}\left(1 / M_{l}^{2}-1\right) r d r$ if this integral exists (i.e., if $\sigma<1 / 2$ ). Since the axial velocity perturbation associated with the $V$ above is 


$$
U \simeq-\frac{\bar{\pi}}{\gamma p^{*}}\left(\frac{T_{w}}{\alpha} \frac{1-\sigma}{1-2 \sigma}(1-r)^{-\sigma}-\alpha \sigma C(1-r)^{\sigma-1}\right)
$$

for $(1-r)<1$, the solution with $C=0$ for $\sigma<1 / 2$ has the weakest possible singularity at the wall. As we shall see next, this is precisely the matching condition imposed by the boundary layer near the wall, and thus it plays the same role for an infinite shear flow with $\sigma<1 / 2$ as the previous condition (7) for a finite shear flow. (Note also that $V=0$ at the wall even if $C$ were different from zero.)

We turn now to consider the viscous boundary layer near the wall. The balance of inertia and viscous forces in the momentum equation (2), with $u=O\left[(1-r)^{\circ}\right]$, yields $(1-r)=O\left[(l-x)^{1 /(\sigma+2)}\right]$ in the boundary layer. In addition, advancing that the pressure gradient does also play a role in the boundary-layer flow, we find the relation $\delta=2 \sigma /(\sigma+2)$ between the two unknown exponents $\delta$ and $\sigma$ introduced before. The density and temperature in the layer differ very little from $\rho_{w}=\rho_{l}(1)$ and $T_{w}$, and therefore compressibility effects are negligible. Curvature effects are also negligible, and the inner flow can be described in terms of a streamfunction $\Psi$, with $\rho_{w} u=-\Psi_{r}$ and $\rho_{w} v=\Psi_{x}$. Accounting for the matching with the outer inviscid flow, the inner solution must be of the form

$$
\begin{gathered}
\Psi=(l-x)^{(\sigma+1) /(\sigma+2)} \beta f(\eta), \\
\text { where } \eta=\beta \frac{1-r}{(l-x)^{1 /(\sigma+2)},}
\end{gathered}
$$

and $\beta=[2 \sigma /(\sigma+2)]^{1 / 4}\left(\rho_{w} \bar{\pi} / \gamma\right)^{1 / 4}$. The momentum equation becomes

$$
f^{\prime \prime \prime}+\frac{\sigma}{\sigma+2} f^{\prime 2}-\frac{\sigma+1}{\sigma+2} f f^{\prime \prime}+1=0,
$$

with the boundary conditions

$$
\begin{aligned}
& f=f^{\prime}=0 \text { at } \eta=0, \\
& f \rightarrow \bar{\alpha} \eta^{\sigma+1} /(\sigma+1) \text { for } \eta \rightarrow \infty,
\end{aligned}
$$

where $\bar{\alpha}=\rho_{w} \alpha / \beta^{\sigma+2}$. The last term of (13) comes from the pressure gradient in (2).

As can be verified, the inner problem (13)-(15) has no solution with $f^{\prime \prime}(0) \geqslant 0$ and $\bar{\alpha}>0$ for $\sigma>1 / 2$, whereas for $0<\sigma<1 / 2$ there is a unique value of $f^{\prime \prime}(0)$ leading to the potential behavior (15), and thus there is a unique solution of (13)-(15) for each $\sigma$. For $\eta>1$, the corresponding axial velocity, written in outer variables $(x, r)$, is

$$
\begin{aligned}
u \sim \alpha(1-r)^{\sigma}-\frac{1-\sigma}{1-2 \sigma} \frac{\bar{\pi} T_{w}}{\gamma p^{*} \alpha}(l-x)^{2 \sigma /(\sigma+2)}(1-r)^{-\sigma} \\
+\frac{\sigma b}{\rho_{w}} \beta^{\sigma+1}(l-x)^{1 /(\sigma+2)}(1-r)^{\sigma-1}+\cdots,
\end{aligned}
$$

where $\bar{\alpha}=\bar{\alpha}(\sigma)$ and $b=b(\sigma)$ only. The first term of (16) matches the limiting velocity $u_{l}(r)$, and the second term, which is due to the pressure gradient in (13), matches the part of the outer velocity (11) proportional to $(1-r)^{-\sigma}$.
Since $(l-x)^{2 \sigma /(\sigma+2)}>(l-x)^{1 /(\sigma+2)}$ for $(l-x)<1$ and $\sigma<1 / 2$, matching requires $C=0$ in (11), as advanced before.

Moreover, matching also implies that the third term of (16), proportional to $b$, must be zero. For, if $b \neq 0$, a term of order $(I-x)^{1 /(\sigma+2)}$ would be induced in the outer expansion, which would obey the same linear equation (9) and regularity condition at the axis as the leading term (8) of the outer expansion. Thus the new term would be merely proportional to the leading term, and its asymptotic behavior for $r \rightarrow 1$, given by (11) with $C=0$, cannot be matched to $(16)$. The condition $b(\sigma)=0$ is verified for $\sigma=\sigma_{c} \simeq 0.364$, for which value $\delta \simeq 0.308$; two other quantities of interest from the numerical solution of (13)-(15) with $\sigma=\sigma_{c}$ are $f^{\prime \prime}(0) \simeq 2.402$ and $\bar{\alpha} \simeq 3.698$. This completes the determination of the flow structure near the critical section.

The nondimensional pressure gradient and wall shear stress for $(l-x)<1$, obtained from the $x$ derivative of the first equation (8) and the second $r$ derivative of (12) at $r=1$, respectively, are

$$
\frac{d p}{d x} \simeq-\frac{0.308 \bar{\pi}}{(l-x)^{0.692}} \quad \text { and } \quad \tau \simeq \frac{0.993 \bar{\pi}^{3 / 4}}{\gamma^{3 / 4} \rho_{w}^{1 / 4}(l-x)^{0.269}},
$$

where the constants $\rho_{w}$ and $\bar{\pi}$ (or, equivalently, $\alpha$ ) depend on the upstream evolution and cannot be determined from the local analysis.

The results of the asymptotic analysis and the numerical solution of (1)-(6) are compared in the excerpt of Fig. 1. This is a logarithmic plot of the pressure gradient versus the wall shear stress obtained from the numerical solution for the three values of $M(0)$ mentioned before [the three lines practically coincide in the range of $(l-x)$ corresponding to this excerpt]. The dashed straight line has slope $0.692 / 0.269=2.572$, which corresponds to eliminating $(1-x)$ between the two equations in (17) (the vertical location of this line is arbitrary).

We may now summarize the two essential ingredients of the analysis: (i) Upstream information propagation in a nonuniform quasi-one-directional flow with regions of low velocity playing a part in the propagation process can only be avoided if the solution develops a singularity. (ii) The nature and strength of this singularity is determined by a local analysis, resulting from the matching of the two regions into which the flow structure splits on approaching the singular section. Since nonuniform flows with the above properties are fairly common, the present analysis has probably a range of applicability wider than the particular flow discussed here.

\footnotetext{
${ }^{1}$ E. Pérez, Ph.D. thesis, Polytechnic University of Madrid, 1989.

${ }^{2}$ A. Bernstein, W. H. Heiser, and C. Hevenor, "Compound-compressible nozzle flow," J. Appl. Mech. 34, 548 (1967).

${ }^{3}$ E. Fage, "Apparent subsonic choking dual-stream nozzles," AIAA J. 14, 681 (1976).

${ }^{4} E$. Fage, R. Marchal, and P. Servanty, "Sur les phénomènes de blocage dans les tuyères multi-flux avec and sans effect de viscosité," L'Aeronaut. L'Astronaut. 105, 54 (1984).
} 